\title{
ON THE EXTENSIVE DERIVATIVE
}

\author{
R. H. BOWMAN
}

I. Introduction. The purpose of the present paper is to extend the concept of extensive differentiation defined by H. V. Craig [2] for the extensors $T_{b}^{\alpha a}$ and $T_{\alpha a}^{b}$. We first note that the extensive derivative in both cases was obtained by intrinsic differentiation of these extensors contracted with $V_{\alpha a}\left[=\left(\begin{array}{c}M \\ A\end{array}\right) V_{a}^{(M-A)}\right]$ and $V^{\alpha a}\left[=V^{a(\alpha)}\right]$, respectively, factoring $V_{\alpha a}^{*}$ and $V^{* \alpha a}$ of range one greater than the original and utilizing the extensor quotient law to fix the extensor character of the remainder, the extensive derivative. Recalling that in the case of scalars intrinsic differentiation reduces to ordinary priming, we adopt the same procedure to define the extensive derivative of any extensor. In III several special cases are treated, and it is seen that only those extensors without tensor indices need be considered. In IV the general definition of the extensive derivative is given and is utilized to prove that if a class $C^{\prime}$ extensor is defined along a suitable arc, then it has a unique extensive derivative.

Use is made of extensive differentiation at various places in the literature [5], [6], [7], and in particular we take note of the fact that the extensor character of some of our derived expressions was established by Kawaguchi in a different way [8]. However, he apparently did not note that they were special cases of a general differentiation process.

II. Notation. The notation employed in this study is essentially that of previous works on the subject (see [2], [3], [4]). Repeated lower case Latin indices are to be summed 1 to $N$, while repeated lower case Greek indices are to be summed (unless the contrary is stated) from zero to some integral value. In this paper plain symbols with repeated lower case Greek indices are to be summed to $M$ while those with a vertical bar preceding the indices, a star, or \#, are summed to $M+1$. Thus, $T_{c}^{\alpha a} U_{\alpha a}$ is to be summed 0 to $M$ on the Greek indices; while $\left.T\right|_{c} ^{\alpha a} U_{\alpha a}^{*}$ and $\left.T\right|_{\alpha a} ^{c} V^{\sharp \alpha a}$ are summed to $M+1$. Also, we adopt the convention that repeated capital Greek indices do not generate sums unless a summation sign is present. Extensors will be described in terms of an ordered 5-tuple of numbers, e.g. $(1,0,2,1,0)$ which respectively refer to the excontravariant, excovariant, contravariant, covariant orders, and the weight. Finally, extensor com-

Received by the editors March 8, 1967. 
ponent symbols with out-of-range Greek indices will be taken to have the numerical value zero. Thus, for example, $T^{-1 . a}=0$ and $T_{M+1 . a}=0$.

III. Special cases. Suppose $\gamma$ is a class $C^{M+1}$ arc of a Riemannian manifold $R_{n}$, and that class $C^{\prime}$ extensor components $T_{c}^{\alpha a}, T_{\alpha a}^{c}$ are defined along it. If in addition extensor components

$$
V^{\alpha a}\left[=V^{a(\alpha)}\right] \quad \text { and } \quad U_{\alpha a}\left[=\left(\begin{array}{c}
M \\
A
\end{array}\right) U_{a}^{(M-A)}\right]
$$

constructed from arbitrary $V^{a}$ 's and $U_{a}$ 's are defined along $\gamma$, we can as in [2] define extensive derivatives for the quantities $T_{c}^{\alpha a}$ and $T_{\alpha a}^{c}$.

Since $V^{\alpha a} T_{\alpha a}^{c}$ are contravariant tensor components, we have, taking their intrinsic derivative,

$$
\delta\left(V^{\alpha a} T_{\alpha a}^{c}\right)=V^{\alpha+1 \cdot a} T_{\alpha a}^{c}+V^{\alpha a} T_{\alpha a}^{c,}+V^{\alpha a} T_{\alpha a}^{d}\left\{\begin{array}{l}
c \\
d
\end{array}\right\}
$$

where

$$
\left\{\begin{array}{l}
c \\
d
\end{array}\right\}=\left\{\begin{array}{l}
c \\
d e
\end{array}\right\} x^{\prime e}
$$

Letting $\alpha=A-1$ and summing 1 to $M+1$ in the first term we have

$$
\delta\left(V^{\alpha a} T_{\alpha a}^{c}\right)=\sum_{A=1}^{M+1} V^{A a} T_{A-1 \cdot a}^{c}+V^{\alpha a} T_{\alpha a}^{c}{ }^{\prime}+V^{\alpha a} T_{\alpha a}^{d}\left\{\begin{array}{l}
c \\
d
\end{array}\right\}
$$

Since symbols bearing out of range doublets are taken as zero, we increase the range in each term 0 to $M+1$ and thus

$$
\delta\left(V^{\alpha a} T_{\alpha a}^{c}\right)=\sum_{\alpha=0}^{M+1} V^{\alpha a}\left(T_{\alpha-1 \cdot a}^{c}+T_{\alpha a}^{c}+T_{\alpha a}^{d}\left\{\begin{array}{l}
c \\
d
\end{array}\right\}\right) .
$$

If we adopt the notation

$$
\left.T\right|_{\alpha a} ^{c} \equiv T_{\alpha a}^{c \prime}+T_{\alpha-1 \cdot a}^{c}+T_{\alpha a}^{d}\left\{\begin{array}{l}
c \\
d
\end{array}\right\}
$$

and observe that the $V^{\alpha a}$ 's are sufficiently arbitrary, we have from the extensor quotient law that $\left.T\right|_{\alpha a} ^{c}$ is an extensor of type $(0,1,1,0,0)$ with range one greater than that of $T_{\alpha a}^{c}$. Thus we take (3.4) as the defining equation of the extensive derivative for extensors of type $(0,1,1,0,0)$.

In the same manner we take the intrinsic derivative of the tensor 
components $U_{\alpha a} T_{c}^{\alpha a}$ and obtain

(3.5) $\left.T\right|_{c} ^{\alpha a} \equiv \frac{M+1-A}{M+1} T^{A a}+\frac{A}{M+1}\left(T_{c}^{A-1 \cdot a \prime}-T_{b}^{A-1 \cdot a}\left\{\begin{array}{l}b \\ c\end{array}\right\}\right)$,

the extensive derivative for extensors of type $(1,0,0,1,0)$ where the range of $\alpha$ is again one greater than that of $T_{c}^{\alpha a}$.

In equations (3.4) and (3.5) a cursory inspection reveals a notational difficulty. The Greek letter $\alpha$ in these equations has the range 0 to $M+1$, while at the outset $\alpha$ had the range 0 to $M$. This inconsistency occurred when we forced an increase in the range, and defined the out of range symbols as zero, in order to have one equation of range 0 to $M+1$ rather than separate equations for the range $\alpha: 0$ to $M$ and $\alpha=M+1$. Hence, we shall depart briefly from the work at hand and introduce a device to correct this inconsistency.

Suppose $E_{\alpha a}$ is an extensor such that the range of $\alpha$ is 0 to $M+1$ if set the tensor rank equal to zero, i.e. $E_{M+1 \cdot a}=0$, it vanishes in all coordinate systems, and the transformation law reduces to that of a range $M$ extensor. Conversely, we may think of an extensor $E_{\alpha a}$ of range $M$ as an extensor of range $M+1$ whose tensor rank is zero, i.e. we may define

$$
E_{\alpha a}^{\#} \equiv \begin{cases}E_{\alpha a} & \text { if } \alpha: 0 \text { to } M \\ 0 & \text { if } \alpha=M+1\end{cases}
$$

In the excontravariant case the situation is a bit more complicated. Here the tensor rank is the zero rank and we cannot add a factor of zero on to the end as before. However, we can construct a range $M+1$ extensor from one of range $M$ as follows. If $D^{p r}=D^{\alpha a} X_{\alpha a}^{p r}$, we may boost the rank by letting $\rho=P-1$ and $\alpha=A-1$, apply the definition of $X_{A-1 . a}^{P-1 . r}$ and obtain

$$
P D^{P-1 \cdot r}=\sum_{A=1}^{M+1} A D^{A-1 \cdot a} X_{A \sigma}^{P r} .
$$

Thus, letting $D^{-1 . a}=0$ we have a range 0 to $M+1$ extensor whose tensor rank is zero. Dividing (3.7) by $M+1$ we may define

$$
\begin{aligned}
& D^{\sharp \alpha a} \equiv \begin{cases}\frac{A}{M+1} D^{A-1 \cdot a} & \text { if } \alpha: 1 \text { to } M+1, \\
0 & \text { if } \alpha=0 \text { and }\end{cases} \\
& D^{\alpha a}=\frac{M+1}{A+1} D^{\#_{A+1 \cdot a}}
\end{aligned}
$$

in the higher order cases a \# will indicate that each of the uncon- 
tracted indices have been adjusted in this manner, e.g.

$$
T^{\# \alpha a \beta b}=\left\{\begin{array}{cl}
A B /(M+1)^{2} T^{A-1 \cdot a B-1 \cdot b} & \text { if } \alpha \text { and } \beta: 1 \text { to } M+1, \\
0 & \text { if } \alpha=0 \text { or } \beta=0 .
\end{array}\right.
$$

Thus (3.4) and (3.5) become

$$
\begin{aligned}
& \left.T\right|_{\alpha a} ^{c}=T_{\alpha a}^{\sharp c,}+T_{\alpha-1 \cdot a}^{\sharp_{c}}+T_{\alpha a}^{\sharp_{a}}\left\{\begin{array}{l}
c \\
d
\end{array}\right\}, \\
& \left.T\right|_{c} ^{\alpha a}=T_{c}^{\sharp A a \prime}+\frac{M+1-A}{A+1} T_{c}^{\# A+1 \cdot a}-T_{b}^{\#_{A a}}\left\{\begin{array}{l}
b \\
c
\end{array}\right\} .
\end{aligned}
$$

If we take the intrinsic derivative of a tensor, we prime the tensor and add or subtract a Christoffel term for each tensor index. And since we have obtained the above derivatives by contracting the extensor index with another extensor and taking the intrinsic derivative of the tensor so obtained, we see that the result is the derivative of the contraction and a Christoffel term for each tensor index.

If we follow this procedure in obtaining extensive derivatives for higher order extensors, we see that the only difference in the extensive derivatives of two extensors of the same extensor type, one without tensor indices and one with tensor indices, will be in the addition of Christoffel terms, exactly as in the case of intrinsic differentiation, for an extensor with tensor indices. Thus, it is only necessary to obtain the extensive derivative of pure extensors. In order to obtain a clearer picture of the emerging pattern we shall investigate the three second order cases $T_{\beta b}^{\alpha a}, T_{\alpha a \beta b}$, and $T^{\alpha a \beta b}$.

Suppose that class $C^{\prime}$ extensor components $T_{\beta b}^{\alpha a}, T_{\alpha a \beta b}$, and $T^{\alpha a \beta b}$ are defined along a class $C^{M+1} \operatorname{arc}$ of $R_{n}$. If in addition extensor components $V^{\alpha a}, V_{\alpha a}$ and $U^{\alpha a}, U_{\alpha a}$ are defined along $\gamma$ as before we consider the derivatives of the complete contractions. Priming $T_{\beta b}^{\alpha a} V^{\beta b} U_{\alpha a}$, letting $\alpha=A-1$ in the term containing $T_{\beta b}^{\alpha a^{\prime}}$ and $\beta=B-1$ in the term containing $V^{\beta+1 \cdot b}$ with the summation in these terms now 1 to $M+1$, applying equations

$$
U_{\alpha a}^{\prime}=\frac{M+1-A}{M+1} U_{A a}^{*}, \quad U_{\alpha-1 \cdot a}=\frac{A}{M+1} U_{A a}^{*}
$$

$$
\text { (where }{ }^{*} \text { indicates the range } 0 \text { to } M+1 \text { ), }
$$

and taking the summation in each of the resulting terms 0 to $M+1$ as before, we obtain 


$$
\begin{aligned}
& \left(T_{\beta b}^{\alpha a} U_{\alpha a} V^{\beta b}\right)^{\prime}=\sum_{A, B=0}^{M+1} U_{A a}^{*} V^{B b} \\
& \quad \cdot\left(\frac{A}{M+1} T_{B b}^{A-1 \cdot a^{\prime}}+\frac{M+1-A}{M+1} T_{B b}^{A a}+\frac{A}{M+1} T_{B-1 \cdot b}^{A-1 \cdot a}\right) .
\end{aligned}
$$

Adopting the notation

$$
\begin{aligned}
\left.T\right|_{\beta b} ^{\alpha a} & \equiv \frac{A}{M+1} T_{B b}^{A-1 \cdot s^{\prime}}+\frac{M+1-A}{M+1} T_{B b}^{A a}+\frac{A}{M+1} T_{B-1 \cdot b}^{A-1 \cdot a} \\
& =T_{B b}^{\# A a^{\prime}}+\frac{M+1-A}{A+1} T_{B b}^{\sharp_{A+1} \cdot a}+T_{B-1 \cdot b}^{\sharp_{A a}}
\end{aligned}
$$

In the same manner we see that

$$
\begin{aligned}
& \left.T\right|_{\alpha a \beta b}=T_{\alpha a \beta b}^{\#}+T_{\alpha-1 \cdot a \beta b}^{\sharp \sharp}+T_{\alpha a \beta-1 \cdot b}^{\#}, \\
& \left.T\right|^{\alpha a \beta b}=T^{\# A a B b^{\prime}}+\frac{M+1-A}{A+1} T^{\# A+1 \cdot a B b}+\frac{M+1-B}{B+1} T^{\#_{A a B+1} \cdot b} .
\end{aligned}
$$

Thus to obtain the extensive derivative in each of these cases, write down the original extensor primed and with \# above, for each excovariant doublet add a term with \# above with that doublet moved away one from tensor rank, e.g. $\alpha a$ becomes $\alpha-1 \cdot a$ for each excontravariant doublet add a term with $\#$ above multiplied by a factor $(M+1-A /(A+1)$ if the Greek index is $\alpha)$ with that doublet moved away one from tensor rank, e.g. $\alpha a$ becomes $A+1 \cdot a$, and for each tensor index we add or subtract a term exactly as in intrinsic differentiation.

IV. The general derivative. In the previous section the concept of extensive differentiation was extended to certain special cases; this was accomplished by contracting the given extensor with certain extensor quantities, differentiating this contraction intrinsically, and factoring the result. We shall utilize this method to define intrinsic differentiation for arbitrary extensors.

Definition. The statement that an extensor of type $\left(Q_{1}, Q_{2}, Q_{3}\right.$, $\left.Q_{4}, 0\right)$ and range $M+1$ is the extensive derivative of an extensor of the same type and range $M$ means that its complete extensor contraction with extensors of the form $U_{\alpha a}^{*}$ and $V^{* \alpha a}$ constructed from arbitrary class $C^{M+1}$ tensors $U_{a}$ and $V^{a}$ is equal to the intrinsic derivative of the tensor obtained by contracting the original extensor with the corresponding $U_{\alpha a}$ 's and $V^{\alpha a}$ 's of one lower range. 
TheOREM. If a class $C^{\prime}$ extensor of type $\left(Q_{1}, Q_{2}, Q_{3}, Q_{4}, 0\right)$ and range $M$ is defined along a parameterized arc $\gamma$ of class $C^{M+1}$, then it has an extensive derivative along $\gamma$.

Proof. In order to apply an inductive argument we must first define an order on the set of extensors.

Definition. The statement that the extensor $A$ precedes the extensor $B$ means that $A$ has fewer extensor indices than $B$.

From the discussion in III we see that only those extensors without tensor indices need be considered, and that extensors with one and two extensor indices do have extensive derivatives. Thus proceeding by induction we assume the theorem is false and consider the set $S$ of extensors not having extensive derivatives. Since the theorem holds for the cases of extensors with one and two indices, there must exist an extensor with a smallest number, say $K+1$ of extensor indices $K+1 \geqq 3$ for which the theorem fails and is consequently valid for extensors with $K$ indices. Suppose that the set of extensors with $K+1$ extensor indices for which the theorem fails have an excovariant doublet $\delta d$. Contraction with an extensor $V^{\delta d}$ constructed from class $C^{M+1}$ tensor components $V^{d}$ yields an extensor with $K$ extensor indices, which has an extensive derivative. Thus, if the extensor is of the form $T_{\gamma c \cdots \delta d}^{\alpha a \cdots \beta b}$ we see that

$$
\begin{gathered}
\left.T_{\cdots \delta d}^{\cdots}\right|_{\gamma c \cdots \beta b} ^{\alpha a \cdots \beta b}=\left(T_{\gamma c \cdots \delta d}^{\sharp \alpha a \cdots \beta b} V^{\delta d}\right)^{\prime}+\frac{M+1-A}{A+1} T_{\gamma c \cdots \delta d}^{\not A+1 \cdot a \cdots \beta b} V^{\delta \alpha} \\
+\cdots+T_{\gamma-1 \cdot c \cdots \delta d}^{\sharp 4 \alpha a \cdots \beta b} V^{\delta d}+\cdots
\end{gathered}
$$

Expanding the first term, letting $\delta=\delta^{\prime}-1$ with summation 1 to $M+1$ in the term containing $V^{\delta \prime+1 \cdot d}$, and taking the summation in each term 0 to $M+1$ and dropping the prime from $\delta^{\prime}$ we have

$$
\left.T \cdots \delta \delta V^{\delta d}\right|_{\gamma c \cdots \beta b} ^{\alpha a \cdots \beta b}=\sum_{\delta=0}^{M+1} V^{* \delta d}\left(T_{\gamma c \cdots \delta d}^{\alpha a \cdots \beta b}+T_{\gamma c \cdots \delta-1 \cdot d}^{\alpha a \cdots \beta b}+\cdots\right)
$$

However, from the definition of extensive differentiation, we have

$$
\begin{aligned}
\delta\left[U_{\alpha a} \cdots W_{\beta b} Y^{\gamma c} \cdots\right. & \left.\cdot\left(V^{\delta d} T_{\gamma c}^{\alpha a \cdots \delta b}\right)\right]=\left.T \cdots \delta \delta V^{\delta d}\right|_{\gamma c \cdots \beta b} ^{\alpha a \cdots b} U_{\alpha a}^{*} \cdots W_{\beta b}^{*} Y^{* \gamma o} \\
= & \sum_{A, \cdots, \Delta=0}^{M+1} U_{A a}^{*} \cdots W_{B b}^{*} Y^{* \Gamma c} \cdots V^{* \Delta d} \\
& \cdot\left(T_{\Gamma c \cdots \Delta d}^{f_{A u} \cdots B b}+\frac{M+1-A}{A+1} T_{\Gamma c \cdots \Delta d}^{\# A+1 \cdot a \cdots B b}+\cdots\right) .
\end{aligned}
$$


Thus $T_{\gamma c \cdots \delta d}^{\alpha a \cdots \beta b}$ has an extensive derivative following the pattern established in III. In the case where the extensor has no excovariant extensor index, an analogous proof holds and is thus omitted. Consequently, we conclude that extensors with $K+1$ extensor indices have extensive derivatives, that the set $S$ has no first element, and consequently the validity of the theorem.

The uniqueness of the extensive derivative follows from the above construction and the definition of extensive differentiation.

\section{REFERENCES}

1. H. V. Craig, Vector and tensor analysis, McGraw-Hill, New York, 1943, pp. 251-302.

2. - On the structure of certain tensors, Math. Mag. 21 (1947), 21-29.

3. - On the structure of intrinsic derivatives, Bull. Amer. Math. Soc. 53 (1947), 332-342.

4. H. V. Craig and B. B. Townsend, On certain metric extensors, Pacific J. Math. 3 (1953), 25-46.

5. Y. Katsurada, On the intrinsic derivative in the non-holonomic exsurface, J. Fac. Sci. Hokkaido Univ. Ser. I 12 (1953), 157-162.

6. - Specialization of the theory of a space of higher order. II. On the extended Lie derivative, Tensor 2 (1952), 15-26.

7. - Alcune trasformazioni parallele di varietà algebriche $(H, K)$ di Del Pezzo-Segre, Atti Accad. Naz. Lincei Rend. Cl. Sci. Fis. Mat. Natur. (8) 22 (1957), 719-725.

8. A. Kawaguchi, Die differentialgeometrie höherer Ordnung. I. Erweiterte Koordinatentransformationen und Extensoren, J. Fac. Sci. Hokkaido Univ. 9 (1940), 1-152.

VANDERBILT UNIVERSITY 